TRANSACTIONS OF THE

AMERICAN MATHEMATICAL SOCIETY

Volume 158, Number 1, July 1971

\title{
ISOLATED INVARIANT SETS AND ISOLATING BLOCKS
}

\author{
BY \\ C. CONLEY( $\left.{ }^{1}\right)$ AND R. EASTON $\left({ }^{2}\right)$
}

Introduction. The restricted three body problem has motivated a considerable amount of research in ordinary differential equations; in this instance the motivation comes from the nonplanar problem. However, the dimension, or even the specific form of the equations, is not relevant to the things discussed and so an easier problem is used here as an example.

Namely, consider given two bowls which are connected by a saddle-like trough and the problem of describing how a point mass slides around in this double bowl under the influence of gravity. Assuming the energy given the point mass is enough that it can go from one bowl to the other, one easily guesses that there are solutions which remain in the trough: if it were too close to either bowl the point mass would fall into the nearer one; somewhere between extremes there should be a way to place it so that it falls into neither.

In fact if the energy is such that there is no place the point mass can rest, one might expect the existence of an unstable periodic orbit in the trough. In the planar restricted three body problem this would correspond to one of the unstable periodic orbits near the collinear Lagrangian point between the masses.

In the nonplanar problem it is not quite so easy to guess the analogue, but a closer look leads one to expect that the role of the periodic orbit is played by a three dimensional sphere of orbits in the five dimensional energy surface. $R$. Sacker [13] has shown there is a smooth such invariant (composed of orbits) three-sphere for each of an interval of values of the energy.

Again one expects the invariant set to be unstable: its existence is surmized on the basis of the instability together with the fact that orbits can leave the vicinity of the invariant set in two essentially different directions-they can fall towards one or the other mass point.

This work concerns a general situation in which such heuristic reasoning can be formalized, see also [6], [8] and [9]. In the intended applications the procedure is to first find a region-like that between the bowls above-wherein one can guess the existence of an invariant set. This region we have called an isolating block; it takes the form of a compact submanifold which has the same dimension as that carrying the flow and which has the property that all orbits tangent to its boundary

Received by the editors April 3, 1969.

(1) Research supported by National Science Foundation under grant GP-8927.

$\left({ }^{2}\right)$ Research supported by United States Air Force under grant AFOSR-67-0693A and by NASA under grant NGR-40-002-015.

Copyright (C) 1971, American Mathematical Society 
bounce off (in both time directions) to the outside. As demonstrated in a different context by $\mathrm{T}$. Ważewski [17] such a property implies that the mapping of the isolating block to the set of outgoing points in the boundary (defined by carrying points forward by the flow) is a strong deformation retraction. In the present situation use is made of this retraction together with that from the block to the set of incoming points defined by following the flow backward in time.

The phrase above that "there are two essentially different space directions away from the set" is reflected in the way the sets of incoming and outgoing points are situated in the boundary of the isolating block.

Using the latter information (rather homological relations between these subsets of the boundary and the block itself) information concerning the cohomology of the maximal invariant set inside the block is derived. In the example of the nonplanar restricted problem we would prove not that the invariant set is a sphere, but that the third Čech cohomology of the invariant set is nontrivial.

The results are thus weak; it is clear that this must be so since only properties of the flow which can be seen at the boundary of the isolating block are used. However gaining even such weak information can be a useful step towards an overall qualitative picture of the flow. In [2] and [10] examples are considered wherein the method gives some not immediately apparent information which aids in picturing the total flow. The treatment is brief in these articles; by combining the present crude techniques with more analytical ones, more detailed results might be proved. (Another example of a somewhat different use of an isolating blockcalled submanifold convex to the flow there-is in [3].)

In addition to the invariant set in the isolating block, the set of those points of the boundary which enter the block and never again leave is partially described in terms of its cohomology. These points must tend to the invariant set since it is maximal in the blocks, so contains all $\omega$-limit sets in the block. Hence they are termed asymptotic, as are those in the set of leaving points which have never entered. As done in [4] and [5] for a very simple case one might discuss properties of the flow which are consequences of the way the flow maps the set of outgoing points to the incoming ones (both sets comprise surfaces of section). In particular the homoclinic point theory of Poincaré and Birkhoff ([1], [14]) is likely to have some analogue in certain cases.

One important aspect of the homoclinic point theory is that the conclusions (in the nondegenerate situation) hold for nearby flows. This depends on the fact that the invariant set as well as the asymptotic sets are stable under perturbation. In the present situation this is replaced by the statement that the isolating block is stable (Theorem 1.6). This does not imply the invariant set is; however those properties of its which can be derived from boundary information alone must also be properties of the invariant set inside the block for the perturbed flow.

We have called the maximal invariant set inside an isolating block "isolated" (cf. [16]) because there is no other invariant set between it and a neighborhood 
of it. Isolated from above might be better, but no use is made here of the complementary notion. Given the isolating block, its interior is such a neighborhood (orbits touching the boundary leave in one or the other or both directions so cannot be in an invariant set inside the block).

The main theorem of this report is the converse statement (1.5); namely any invariant set that is the maximal one in some neighborhood of itself is the maximal one in some isolating block. This result together with the perturbation result and the fact that the form of the block determines properties of the invariant set indicate that a useful step in the qualitative analysis of flows might be to search for isolated invariant sets.

We do not say much about the existence (in general) of isolated invariant sets; later it will be shown that in the space of closed invariant sets with the Hausdorff metric they correspond to sets which are both closed and open. Thus if they do not exist, the space of closed invariant sets is a continuum. (A component in this space corresponds to the notion of a quasi-isolated invariant set, namely one which is the intersection of the isolated invariant sets containing it. This notion will also be discussed later.)

Some statement concerning the setting should be given: We have discussed smooth flows on manifolds, but it soon becomes clear that this setting is unnatural to the topic. Much of the work (including the block theorem, 1:5, with an appropriately modified definition) could be carried out for flows on compact metric spaces. The perturbation theorem should then be proved with the compactopen topology on flows. However the details are less transparent; convenient use has been made of things like the duality theorem for manifolds and the stability of the notion of a surface of section. Also proofs have been sketched which are not quite so clear in the more general framework.

One result which does depend on the manifold setting is this: if the isolated invariant set has finitely generated cohomology, then the asymptotic sets do also. This follows because manifolds have finitely generated homology. (Most of the significance of this theorem is revealed if one proves it directly for a rest point in the plane isolated by a disk of which it is the center. If an orbit is tangent to the boundary of the disk it must go away from the disk in both time directions-in particular it cannot be a rest point. One sees that the set of points in the boundary of the disk which enter and never come out again must have a finite number of components.)

In $\S 1$ definitions are given and the two basic theorems are stated; the proofs are deferred to $\S 4$ and $\S 5$. In $\S 2$ theorems are proved which show how properties of the invariant set may be derived from those of the isolating block. In $\S 3$ some (rather artificial) examples are discussed.

In $\$ 2$ and $\$ 3$ some elementary algebraic topology is used; E. H. Spanier's book, [15] has been our reference here. Since our main desire has been to see that results of possible interest in differential equations might be obtained using the notion 
of isolating blocks, it was a great pleasure to find a book which makes available in such lucid form all the theorems (and more) which we guessed might be used to illustrate our point. In the future we hope to develop this aspect of the paper to the point where it is something more than just elementary applications of well-known theorems from algebraic topology; in any case Spanier's book has made the initial stages of that job very pleasant.

\section{Notation, definitions, and two basic theorems.}

A. Standing notation. Let $V$ denote a smooth $\left(C^{\prime}\right)$ vector field on a smooth manifold $M$ and let $R$ denote the real numbers. Then $V$ defines a smooth flow on $M$ which we will denote $\varphi_{V}: M \times R \rightarrow M$. When $V$ is understood, we let $p \cdot t$ denote $\varphi_{V}(p, t)$; also for $A \subset M$ and $T \subset R, A \cdot T \equiv\{p \cdot t \mid p \in A, t \in T\}$.

An invariant set of $\varphi_{V}$ is a set $I \subset M$ such that $I \cdot R=I$; generally we discuss only closed invariant sets.

Other standing notation is as follows: $R^{+}$denotes the nonnegative reals, $R^{-}$ the nonpositive ones; for $a, b \in R,(a, b)$ denotes the open interval from $a$ to $b$ and $[a, b]$ the closed one; finally the closure and boundary of a set $S$ in a topological space are denoted $\mathrm{Cl}(S)$ and $\partial S$ respectively.

B. Isolated invariant sets.

1.1 Definition. An isolating neighborhood for the flow $\varphi_{V}$ on $M$ is an open set $U \subset M$ such that $p \in \partial U$ implies $p \cdot R \notin \mathrm{Cl}(U)$.

Observe that since points of $\partial U$ leave $\mathrm{Cl}(U)$, the maximal invariant set of $\varphi_{V}$ in $U$ must be closed and is also the maximal invariant set in $\mathrm{Cl}(U)$.

1.2. Definition. An invariant set $I$ is called isolated if $I$ is the maximal invariant set in some isolating neighborhood $U$. In this case say $U$ is an isolating neighborhood of $I$.

Observe that isolated invariant sets are closed, and that if $U$ is an isolating neighborhood of $I$, then any open set between $U$ and $I$ is also. The word isolated refers to the fact that there are no invariant sets between $I$ and $\mathrm{Cl}(U)$.

As examples of isolated invariant sets we cite elementary orbits (critical points and periodic solutions all of whose eigenvalues have modulus different from one) and attracting and repelling sets. Note that an isolated critical point is not necessarily isolated as an invariant set; in fact in two dimensions, those that are must have index less than or equal to one. A somewhat less simple example arises in the study of nondegenerate homoclinic points [1], [14]. In the latter reference, S. Smale shows that the mapping associated with such points admits an invariant Cantor set, $C$, on which it is equivalent to the shift automorphism. In terms of the flow, this invariant set corresponds to the product of $C$ with an interval where the ends are identified by the shift; this "Cantor cylinder" of orbits is an isolated invariant set. Other examples are given later.

C. Isolating blocks. An isolating block for the flow $\varphi_{V}$ on $M$ is a compact submanifold (with boundary) of $M$ with the same dimension as $M$ and such that the 
vector field $V$ behaves suitably on the boundary, in particular the interior is an isolating neighborhood.

1.3. Definition (Notation). Let $N \subset M$ be a smooth compact submanifold (with boundary) of $M$ with $\operatorname{dim} N=\operatorname{dim} M$, and let $\partial N=n$. Define

$$
\begin{aligned}
n^{+} & \equiv\{p \in n \mid \exists \varepsilon>0 \text { with } p \cdot(-\varepsilon, 0) \cap N=\varnothing\}, \\
n^{-} & \equiv\{p \in n \mid \exists \varepsilon>0 \text { with } p \cdot(0, \varepsilon) \cap N=\varnothing\}, \\
\tau & \equiv\{p \in n \mid V \text { is tangent to } n \text { at } p\} .
\end{aligned}
$$

In general $n^{+}, n^{-}$and $\tau$ might be variously related but their union must always be $n$ : points of $n^{+}$leave $N$ going backwards, those in $n^{-}$leave $n$ going forwards and the remainder must be in $\tau$. We are interested in the case where $n^{+} \cap n^{-}=\tau$; in particular all points of $n=\partial N$ leave $N$ in one or the other direction-both for $\tau$ -so that the interior of $N$ is an isolating neighborhood for $\varphi_{V}$.

With one technical requirement we have the definition of an isolating block:

1.4 Definition. With the notation of $1.3, N$ is an isolating block for $\varphi_{V}$ if $n^{+} \cap n^{-}=\tau$, and if $\tau$ is a smooth submanifold of $n$ with codimension one and (as a consequence) $n^{+}$and $n^{-}$are submanifolds with common boundary $\tau$.

D. Statements of the two basic theorems. We have seen that the interior of an isolating block is an isolating neighborhood and so determines an isolated invariant set-possibly empty. The first theorem states that any isolated invariant set can be determined in this manner. The proof, consisting of a construction of the block, is given in $§ 5$. The second theorem states that isolating blocks are "stable"; this proof is sketched in $\S 4$. For contrast we also state (and sketch the proof of) a theorem concerning the stability of isolating neighborhoods. This latter theorem contrasts with that for the blocks in that it allows no conclusion to be made concerning the stability of the invariant set while that for the blocks does as we will see in $\S 2$.

1.5 THEOREM. I is an isolated invariant set if and only if it is the maximal invariant set in some isolating block.

1.6 THEOREM. Let $\varphi_{V}$ admit an isolating block $N$. Then there is a neighborhood $\nu$ of $V$ in the $C^{0}$ topology on vector fields such that for any (smooth) $W \in \nu$, the flow $\varphi_{\mathrm{W}}$ admits an isolating block $\tilde{N}$ diffeomorphic to $N$ in such a way as to map $\tilde{n}^{+}$to $n^{+}, \tilde{n}^{-}$to $n^{-}$and $\tilde{\tau}$ to $\tau$. (In fact the diffeomorphism is the restriction of an autodiffeomorphism of $M$.)

The third theorem fits a more general setting:

1.7 THEOREM. Let $U$ be an isolating neighborhood for a (continuous) flow $\varphi: X \times R \rightarrow X$ on the compact metric space $X$ (cf. 1.1).

Then there is a neighborhood $\Phi$ of $\varphi$ in the compact open topology such that $U$ is an isolating neighborhood for all flows $\psi$ in $\Phi$. 
The proof of this theorem follows directly from the definition of an isolating neighborhood, the definition of the compact open topology, and the compactness of $\partial U$. Thus each point $p$ of $\partial U$ admits a compact neighborhood $C_{p}$ which is carried outside of $\mathrm{Cl}(U)$ in finite time $t_{p}$. Furthermore a finite number, say $C_{p_{1}}, \ldots, C_{p_{n}}$ of these neighborhoods cover $\partial U$. The neighborhood $\Phi$ is that determined by the $2 n$ compact sets $C_{p_{i}} \times\{0\}$ and $C_{p_{i}} \times\left\{t_{p_{i}}\right\}$ (in $X \times R$ ) and open sets $W_{p_{i}}$ and $\tilde{W}_{p_{i}}$ (in $\left.X\right)$ such that $W_{p_{i}} \supset \varphi\left(C_{p_{i}}, 0\right)$ and $\tilde{W}_{p_{i}} \supset \varphi\left(C_{p_{i}}, t_{p_{i}}\right)$ and where the $\tilde{W}_{p_{t}}$ do not meet $\mathrm{Cl}(U)$.

The relative strength of the block theorem compared to the neighborhood theorem is illustrated in the next section where we discuss properties of the isolated invariant set in a block which are determined solely from the behavior of $V$ on the boundary of the block. It is clear that 1.6 implies these properties are "stable" whereas 1.7 does not.

2. Cohomology of the isolated invariant set and the asymptotic sets determined by the isolating block.

A. The asymptotic sets.

2.1 Definition. Let $N$ be an isolating block for $\varphi_{V}$. Define

$$
\begin{aligned}
A^{+} & \equiv\left\{p \in N \mid p \cdot R^{+} \subset N\right\}, & A^{-} & \equiv\left\{p \in N \mid p \cdot R^{-} \subset N\right\}, \\
I & \equiv A^{+} \cap A^{-}, & A & \equiv A^{+} \cup A^{-}, \\
a^{+} & \equiv n \cap A^{+}=n^{+} \cap A^{+}, & a^{-} & \equiv n \cap A^{-}=n^{-} \cap A^{-} .
\end{aligned}
$$

A simple example (a hyperbolic critical point in the plane) of an isolating block is drawn in Figure 1. The disk is $N$, and $\tau$ consists of the four distinguished points of the circle, $n$, through which pieces of tangent orbits have been drawn. The set

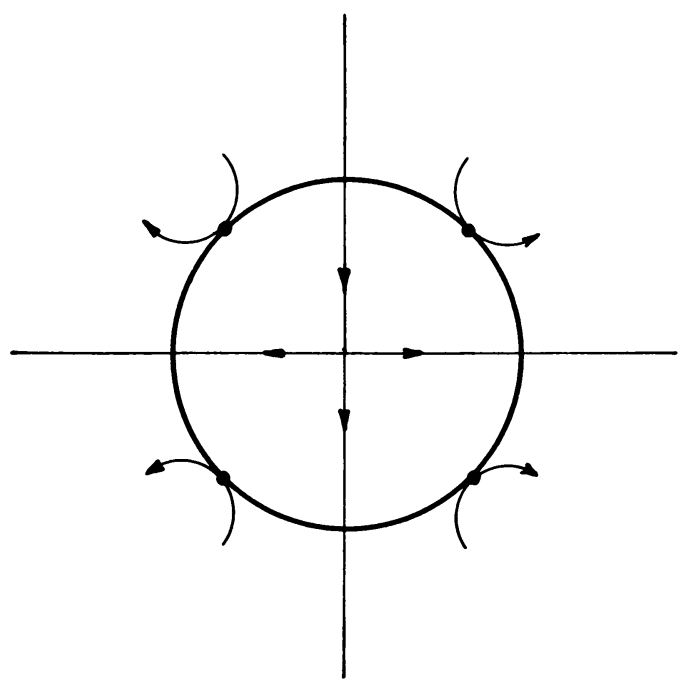

FIGURE 1 
$n^{+}$consists of the top and bottom arcs of $n, n^{-}$those on the sides. The sets $\mathrm{A}^{+}$ and $A^{-}$are (respectively) the vertical and horizontal straight line segments in $N$ and their intersection, the critical point, is $I$. Finally $a^{+}$consists of the endpoints of $A^{+}$in $n, a^{-}$those of $A^{-}$.

An immediate consequence of the definitions $(1.3,1.4,2.1)$ is

2.2 Corollary. $A^{+}, A^{-}, I, a^{+}$and $a^{-}$are compact sets and the latter three are contained in the interior of $N, n^{+}$and $n^{-}$respectively.

B. Deformation retractions defined by the flow. Lemma 2.4 below is the essential consequence of the fact that $N$ is an isolating block and enables us to derive properties of $I, a^{+}$and $a^{-}$from those of $N, n^{+}, n^{-}$and $\tau$. (Recall that $R^{+}$and $R^{-}$ contain 0.)

2.3 Definitions. Define functions $\sigma^{+}$and $\sigma^{-}$from $N$ to the extended real numbers $(\{-\infty\} \cup R \cup\{+\infty\}$ with the topology of the closed interval $)$ by

$$
\begin{aligned}
& \sigma^{+}(p)=\sup \left\{t \in R^{+} \mid p \cdot[0, t] \cap n^{-}=\varnothing\right\}, \\
& \sigma^{-}(p)=\inf \left\{t \in R^{-} \mid p \cdot[t, 0] \cap n^{+}=\varnothing\right\} .
\end{aligned}
$$

Also define $r^{+}:\left(N-A^{+}\right) \times[0,1] \rightarrow N-A^{+}$and $r^{-}:\left(N-A^{-}\right) \times[0,1] \rightarrow N-A^{-}$ by

$$
r^{+}(p, t)=p \cdot\left(t \cdot \sigma^{+}(p)\right), \quad r^{-}(p, t)=p \cdot\left(t \cdot \sigma^{-}(p)\right) .
$$

Observe that if $\sigma^{+}(p)$ is finite-namely if $p \in N-A^{+}$-then $p \cdot \sigma^{+}(p) \cdot t$ is in $N-A^{+}$so $r^{+}$is well defined. Similarly, $r^{-}$is well defined.

Because $N$ is an isolating block we have:

2.4 Lemma. The functions $\sigma^{+}, \sigma^{-}, r^{+}$, and $r^{-}$are continuous and the latter two are strong deformation retractions.

Proof. The statement concerning $r^{+}$follows from the continuity of $\sigma^{+}$on observing that $\sigma^{+} \mid n^{-}$is identically zero (points of $n^{-}$leave $N$ as time increases); $r^{-}$is treated similarly.

To prove $\sigma^{+}$is continuous we first observe that, when finite, $\sigma^{+}(p)$ is the first nonnegative time that the orbit through $p$ leaves $N$ (with increasing time): it is certainly the first time the orbit meets $n^{-}$, and once meeting $n^{-}$it must leave $N$; furthermore, orbits must leave $N$ through $n^{-}$so it cannot have left earlier. In particular there is a positive $\varepsilon$ such that $p \cdot\left(\sigma^{+}(p), \sigma^{+}(p)+\varepsilon\right) \cap N=\varnothing$. Thus if $q_{n} \rightarrow p, q_{n} \cdot\left(\sigma^{+}(p)+\varepsilon\right)$ is eventually outside $N$. It follows that $\lim \sup \sigma^{+}\left(q_{n}\right)$ $\leqq \sigma^{+}(p)+\varepsilon$ and since $\varepsilon$ is arbitrary, $\sigma^{+}$is upper semicontinuous. Also, except in the case when $p$ is in $\tau$, the orbit segment $p \cdot\left[\varepsilon, \sigma^{+}(p)-\varepsilon\right]$ is interior to $N$ for $\varepsilon>0$. The same must then be eventually true for any sequence $q_{n} \rightarrow p$. Consequently $\lim \inf \sigma^{+}\left(q_{n}\right) \geqq \sigma^{+}(p)-\varepsilon$ and $\sigma^{+}$is lower semicontinuous at $p$. Finally, since $\sigma^{+} \geqq 0$, and 0 on $\tau$, it is lower semicontinuous on $\tau$, hence everywhere. Thus $\sigma^{+}$ is continuous. The similar argument proves $\sigma^{-}$is continuous and Lemma 2.4 follows. 
An immediate consequence is:

2.5 CoRollaRy. (1) $n^{+}$and $n^{-}$are strong deformation retracts of $N-A^{-}$and $N-A^{+}$respectively;

(2) $n^{+}-a^{+}$and $n^{-}-a^{-}$are both strong deformation retracts of $N-A$;

(3) $r^{-}(\cdot, 1): n^{+}-a^{+} \rightarrow n^{-}-a^{-}$and $r^{+}(\cdot, 1): n^{-}-a^{-} \rightarrow n^{+}-a^{+}$are homeomorphisms inverse to each other, they are the maps defined in the natural way by the flow.

Another result we rely on in the next paragraph is the following:

2.6 LemMA. The inclusion map $(N-A, n-a) \rightarrow(N-I, n)$ induces isomorphism on singular homology, while the inclusion $I \rightarrow A$ induces isomorphism on Cech cohomology.

Both statements follow if it can be shown that in any neighborhood of $I$ a strong deformation retract of $A$ can be found, so we begin with that.

Let $A_{t} \equiv A^{+} \cdot t \cup A^{-} \cdot(-t)$. Since the set $\bigcap_{t>0} A_{t}$ is an invariant set in $N$ it must be contained in $I$; hence, since it contains $I$, it is equal to $I$. Thus (by compactness) if $U$ is a neighborhood of $I, A_{t}$ is eventually in $U$. Furthermore, it is clear that for any $\sigma>0, \mathrm{Cl}\left(A-A_{\sigma}\right)$ is homeomorphic to $\left(a^{+} \cup a^{-}\right) \times[0, \sigma]$ by the map $(p, t) \rightarrow p \cdot t$. The strong deformation retraction $A$ to $A_{\sigma}$ can be defined in an obvious way using that from $\left(a^{+} \cup a^{-}\right) \times[0, \sigma]$ to $\left(a^{+} \cup a^{-}\right) \times\{\sigma\}$.

Now each inclusion $A_{\sigma} \rightarrow A_{t}(t<\sigma)$ induces isomorphisms on the cohomology map while the direct limit of the inclusion induced homomorphisms $\hat{H}^{*}(I) \rightarrow$ $\hat{H}^{*}\left(A_{\sigma}\right)$ is an isomorphism [15, Theorem 6, p. 318 with Corollary 8, p. 334]; it follows that each one is an isomorphism.

Finally an excision $\left(^{3}\right)$ shows that the inclusion $(N-A, n-a) \rightarrow\left(N-A_{\sigma}, n\right)$ $(\sigma>0)$ induces isomorphism on singular homology and since $A_{\sigma}$ can be pulled into any neighborhood of $I$ the inclusion to $(N-I, n)$ follows.

C. The cohomology of $a^{ \pm}$and I determined by the isolating block. In this paragraph and the sequel we will always assume manifolds have orientations over the coefficient ring used.

This paragraph contains proofs of three theorems which illustrate that some properties of the asymptotic and invariant sets are determined by the form of the isolating block. These are rather weak theorems-namely the conclusions only involve statements about cohomology groups-but it is clear that not much more could be expected in view of the weak hypothesis. Even the cohomology groups are far from determined and it can be seen from Figure 1, say, that this will always be the case. With reference to that figure, we are only assuming that properties of $V$ on the bounding circle are given-clearly the inside can be filled in a countless number of different ways. A sensible question, which we will not treat, asks what

$\left({ }^{3}\right)$ This argument, as well as most of the later ones involving excisions, makes use of a collar on the boundary (of $N$ in this case). 
properties of the invariant set are "generic" (in an appropriate topology). We do give one theorem (2.12) concerning the amount of additional information needed to determine the cohomology of $I$; it may be mildly interesting to know that knowledge of $a^{+}$and $a^{-}$(i.e. complete boundary information) is not enough.

We begin by observing that any flow admitting an isolating block such as that in Figure 1 must have a nontrivial invariant set inside. This follows from 2.5; namely, if $I$ (hence $A$ ) were empty, $n^{+}$would be a strong deformation retraction of $N$-which it is not. (Poincaré's formula for computing the index of a vector field on a circle does more in that it shows the invariant set contains a critical point; on the other hand, it says nothing about the asymptotic set.)

Of course nontriviality of $H_{*}\left(N, n^{+}\right)$or $H_{*}\left(N, n^{-}\right)$must always imply that of the invariant set; Theorem 2.8 is the relevant result. Before doing the theorem we state a lemma of which we make frequent use.

2.7 Lemma. Let $H^{*}$ be singular homology and $\hat{H}^{*}$ be Čech cohomology and let $d=\operatorname{dim} M=\operatorname{dim} N$ :

$$
H_{p}\left(n^{ \pm}, n^{ \pm}-a^{ \pm}\right) \approx \hat{H}^{d-p-1}\left(a^{ \pm}\right) \text {and } H_{p}(N, N-I) \approx \hat{H}^{d-p}(I) .
$$

Proof. This follows from the general duality theorem [15, Theorem 17, p. 296].

2.8 THEOREM. There exists a commutative diagram as displayed below wherein the rows are exact and the vertical maps are induced by inclusions. (All homomorphisms marked $\partial$ lower degree by one.)

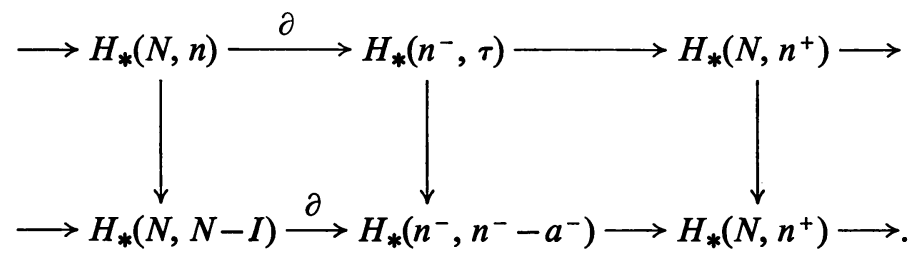

A similar result, obtained by interchanging + and - signs, is true.

Note that it is the bottom row which shows the affect of $H_{*}\left(N, n^{+}\right)$on $\hat{H}^{*}(I)$ and $\hat{H}^{*}\left(a^{-}\right)$.

Proof. The first two columns are portions of the exact sequences for the triples $(N, N-I, n)$ and $\left(n^{-}, n^{-}-a^{-}, \tau\right)$. The bottom two rows are derived from the exact sequences for the triples $(N, n, \tau)$ and $\left(N, N-I, n^{+}\right)$on replacing $H_{*}\left(n, n^{+}\right)$ and $H_{*}\left(N-I, n^{+}\right)$by $H_{*}\left(n^{-}, \tau\right)$ and $H_{*}\left(n^{-}, n^{-}-a^{-}\right)$which is justified as follows:

The diagram below consists of chain maps induced by inclusions:

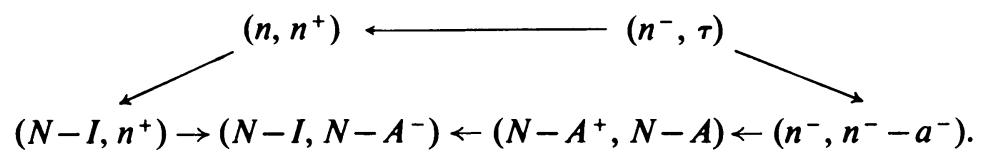


Observe that going from $\left(n^{-}, \tau\right)$ to $\left(N-I, N-A^{-}\right)$by either possible route gives the same result. Furthermore the top horizontal map induces an isomorphism on homology by excision, the first bottom horizontal map induces isomorphism by 2.5 , the second by excision and the last by 2.5 again. From these arguments we now can verify the diagram.

From the bottom row in 2.8 and 2.7 one sees that each cycle in $H_{*}\left(N, n^{+}\right)$ corresponds either to a (class of) cocycles in $H^{*}\left(a^{+}\right)$or a cocycle in $H^{*}(I)$. Using the two rows one sees that those in the image of $H_{*}\left(n^{-}, \tau\right)$ must be of the first sort; however, the others cannot be classified in general (cf. Example 3.3 in $\$ 3 \mathrm{~A}$ ).

It is also of interest to find cycles in $H_{*}\left(n^{-}, \tau\right)$ which go nontrivially to $H_{*}\left(n^{-}, n^{-}-a^{-}\right)$under the inclusion-carriers of these must meet $a^{-}$. With this in mind we state the following corollary of 2.8 .

\subsection{Corollary. Let}

$$
\alpha: H_{*}\left(n^{-}, \tau\right) \rightarrow H_{*}\left(N, n^{+}\right), \quad \alpha^{\prime}: H_{*}\left(n^{-}, n^{-}-a^{-}\right) \rightarrow H_{*}\left(N, n^{+}\right)
$$

and $\beta: H_{*}\left(n^{-}, \tau\right) \rightarrow H_{*}\left(n^{-}, n^{-}-a^{-}\right)$be the homomorphisms in 2.8. Then $\beta$ induces the following injection:

$$
0 \rightarrow H_{*}\left(n^{-}, \tau\right) / \operatorname{ker} \alpha \rightarrow H_{*}\left(n^{-}, n^{-}-a^{-}\right) / \operatorname{ker} \alpha^{\prime} .
$$

$A$ similar statement, obtained on interchanging + and - signs, holds.

The proof just uses the diagram of 2.8 .

As an example we may take the isolating block of Figure 1. Here either of the arcs making up $n^{-}$carry a cycle in $H_{*}\left(n^{-}, \tau\right)$ which goes nontrivially to $H_{*}\left(N, n^{+}\right)$, hence nontrivially to $H_{*}\left(n^{-}, n^{-}-a^{-}\right)$. Thus these arcs must meet $a^{-}$no matter how one fills in the flow.

Theorem 2.8 (and 2.9) give some specific information about $a^{+}$, but not much about $I$ itself. In the theorem below, we make use of the duality isomorphism for manifolds given by taking the slant product with an orientation cocycle. Theorems 17 (p. 289) and 1 (p. 287) of [15] can be applied directly to the double of the manifold (with boundary) $v$ appearing in the statement of 2.10 ; the latter follows on using the appropriate excisions:

2.10 THEOREM. Let $v$ be a submanifold of $n^{+}$with boundary $\partial v$ and with $a^{+} \cap v$ interior to $v$.

The diagram below, in which the two vertical isomorphisms follow from the duality theorem and the other maps are induced by inclusion, is commutative:

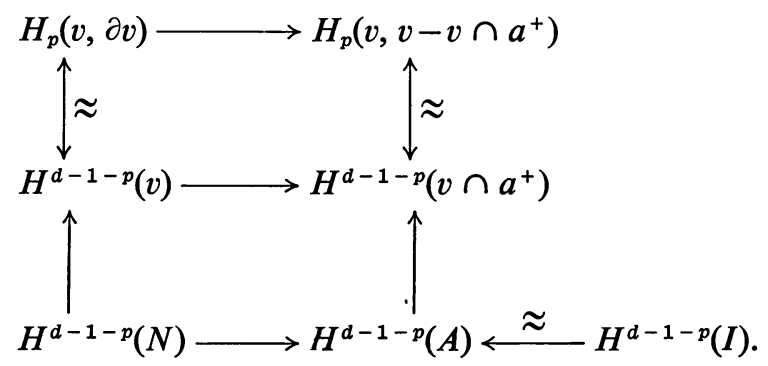


In particular, a sufficient condition that a cocycle of $H^{*}(N)$ goes nontrivially to $H^{*}(I)$ under the inclusion $I \rightarrow N$ is that its image in $H_{p}(v, \partial v)$ goes nontrivially to $H_{p}\left(v, v-v \cap a^{+}\right)$.

The statement obtained by interchanging + and - signs also holds.

As an application we can again consider Figure 1. We have seen that the top arc (say) in $n^{+}$carries a cocycle in $H_{1}\left(n^{+}, \tau\right)$ which goes nontrivially to $H_{1}\left(n^{+}\right.$, $n^{+}-a^{+}$) hence it must meet $a^{+}$. With $v$ equal to this component of $n^{+}$we see that the dual cocycle of the generator of $H_{1}(v, \partial v)$ is the image of the generator of $\hat{H}^{0}(N)$ which must therefore go nontrivially to $\hat{H}^{0}(I)$.

A third theorem which again gives information on $H^{*}(I)$, this time via the cohomology $H^{*}(N, N-I)$, makes use of 2.5 together with the cup product:

2.11 Theorem. Any cocycle $\gamma \in H^{*}(N, n)$ which is the (cup) product of cycles $\alpha \in H^{*}\left(N, n^{+}\right)$and $\beta \in H^{*}\left(N, n^{-}\right)$comes from a cocycle in $H^{*}(N, N-I)$ under the inclusion $(N, n) \rightarrow(N, N-I)$.

This is a direct consequence of 2.5 together with properties of cup products (statement 8 , p. 251 of [15]). Namely, the inclusions $\left(N, n^{+}\right) \rightarrow\left(N, N-A^{-}\right)$and $\left(N, n^{-}\right) \rightarrow\left(N, N-A^{+}\right)$induce cohomology isomorphisms-thus $\alpha$ and $\beta$ are images of cocycles $\alpha^{1}$ and $\beta^{1}$ in $H^{*}\left(N, N-A^{-}\right)$and $H^{*}\left(N, N-A^{+}\right)$respectively and $\gamma$ is then the image of $\alpha^{1} \cup \beta^{1}$ in $H^{*}\left(N, N-A^{+} \cup N-A^{-}\right)=H^{*}(N, N-I)$.

In keeping with tradition, the isolating block of Figure 1 serves as an example: we consider $(N, n)$ to be the product $\left(J^{+}, J^{+}\right) \times\left(J^{-}, J^{-}\right)$where $J^{*}$ is the unit interval, $J^{*}$ the boundary, and where $J^{+} \times J^{-}$corresponds to $\tau$. Then the generators of $H^{1}\left(J^{ \pm}, J^{ \pm}\right)$correspond (via the projections) to those of $H^{1}\left(N, n^{+}\right)$and $H^{1}\left(N, n^{-}\right)$ and their cross product to the cup product of the latter as well as to the generator of $H^{2}(N, n)$ (p. 251, Theorem 7 of [15]).

As remarked already, $H^{*}(I)$ is far from determined by the isolating block. One might ask what additional information is needed. Example 3.3 of the next

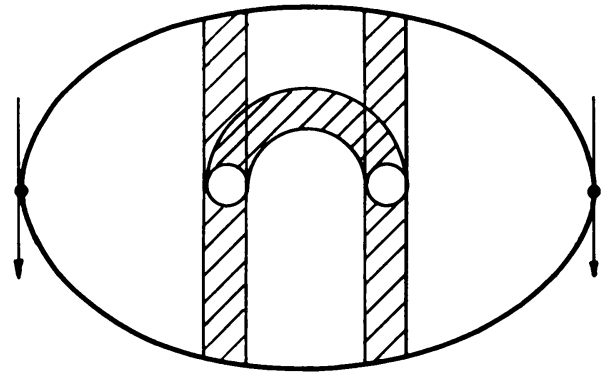

$2 a$

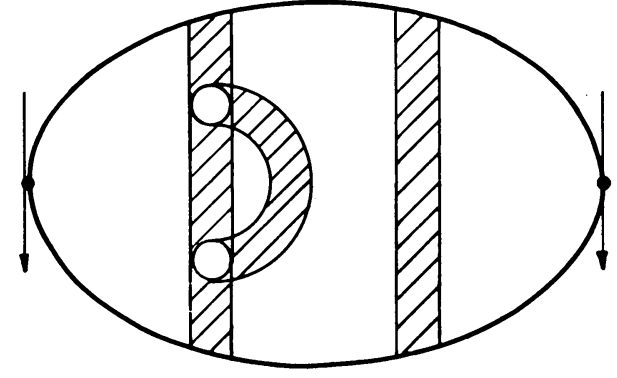

$2 b$

Figure 2. The block $N$ is a disk with a handle; $A$ is shaded in both figures. Recall that $A$ and $I$ have the same cohomology. 
section shows it is not enough to know $a^{+}$and $a^{-}$, and indicates (Figure 3) that the way cycles in $n^{+}-a^{+}$are carried to $n^{-}-a^{-}$might be important since Figures $3 a$ and $3 b$ differ in this respect.

However, even if the homeomorphism from $n^{+}-a^{+}$to $n^{-}-a^{-}$is known, $H^{*}(I)$ is not determined as the pictures below show (Figure 2). In the first picture $I$ has one component, in the second, two. In this case the map from $H_{*}(n) \rightarrow H_{*}(N)$ is not surjective, thus not all of the homology of $N$ is seen from the boundary.

The following theorem rules out such situations:

2.12 THEOREM. Let $H_{*}(N) \rightarrow H_{*}(N, n)$ be trivial and assume $a^{+}$and $a^{-}$as well as the isomorphism $H_{*}\left(n^{+}-a^{+}\right) \stackrel{\varphi}{\rightarrow} H_{*}\left(n^{-}-a^{-}\right)$, defined by the flow, are known.

Then the rank of $H^{*}(I)$ is determined.

For the proof take coefficients in a field. In view of the first hypothesis, the exact sequence for the pair $(N, N-I)$ breaks up into short exact sequences:

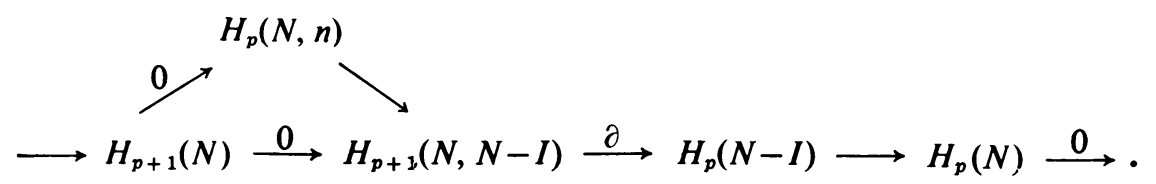

Thus $\operatorname{dim} H_{p+1}(N, N-I)=\operatorname{dim} H_{p}(N-I)-\operatorname{dim} H_{p}(N)$ and we have only to compute $\operatorname{dim} H_{p}(N-I)$ which is done using the Meyer Vietoris Sequence:

$$
\stackrel{\Delta}{\longrightarrow} H_{*}(N-A) \stackrel{i}{\longrightarrow} H_{*}\left(N-A^{+}\right) \oplus H_{*}\left(N-A^{-}\right) \longrightarrow H_{*}(N-I) \stackrel{\Delta}{\longrightarrow} .
$$

Since (2.5), $H_{*}(N-A) \approx H_{*}\left(n^{+}-a^{+}\right) ; H_{*}\left(N-A^{+}\right) \approx H_{*}\left(n^{-}\right)$and $H_{*}\left(N-A^{-}\right)$ $\approx H_{*}\left(n^{+}\right)$, the first two groups in the portion of the sequence shown are known. Now let $i^{ \pm}$denote the (two) inclusion induced homomorphisms from

$$
H_{*}\left(n^{ \pm}-a^{ \pm}\right) \rightarrow H_{*}\left(n^{ \pm}\right) .
$$

It is then easy to see that $i$ (in the sequence) is equivalent to

$$
j: H_{*}\left(n^{+}-a^{+}\right) \rightarrow H_{*}\left(n^{-}\right) \oplus H_{*}\left(n^{+}\right)
$$

where $j=i^{-} \circ \varphi \oplus\left(-i^{+}\right)$. Hence $\varphi$ is given by hypothesis, as are $i^{+}$and $i^{-}$. Thus kernel $i$ is known and $\operatorname{dim} H^{*}(N-I)$ is determined.

We leave this aspect of the paper with the remark that the above collection of theorems is not meant to be complete. We hope those given (with the examples in §3) suffice to illustrate the point intended; namely, that properties of $I$ follow from properties of the block.

3. Some examples. The examples here are artificial; we aim to show later that combinations of these methods with more analytical ones can lead to more interesting results. 
A. Solid tori as isolating blocks. Consider the solid torus as the product of a disk with polar coordinates $\{(r, \theta): 0 \leqq r \leqq 1\}$ and a circle with (angular) coordinate $\psi$.

3.1 EXAMPLE. Let $\tau$ consists of the four circles $\{r=1, \theta=k \pi / 2, k=0,1,2,3\}$ -thus we might be describing an isolating block about a hyperbolic periodic orbit (the "product" of Figure 1 and a circle). The sets $n^{+}$and $n^{-}$then each consist of two annuli. Fixing on one component of $n^{+}$, say $v$, we see that any arc in this component which connects the two bounding circles carries a cycle in $\mathrm{H}_{1}\left(n^{+}, \tau\right)$ which is not in the range of $H_{2}(N, n)$ and so (by 2.9) is carried nontrivially to $H_{1}\left(n^{+}, n^{+}-a^{+}\right)$. This implies $H^{1}\left(v \cap a^{+}\right) \neq \varnothing$, and more geometrically that any arc connecting the two boundary components of $v$ meets $a^{+}$.

Under the duality map this cycle (now viewed in $H_{1}(v, \partial v)$ ) goes to the generator of $H^{1}(v)$ and this latter is the image of that of $H^{1}(N)$. By $2.10 \hat{H}^{1}(N)$ maps nontrivially to $\hat{H}^{1}(I)$. Thus I contains a "Čech circle" going about the hole in the solid torus - as would be expected.

Theorem 2.11 could also be made to apply in this case; the argument is given somewhat more generally in 3.5.

3.2 ExAmple. We might give Example 2.13 a twist by letting the tangency set, $\tau$, consists of the two circles defined by $(r=1$ and) $\theta=\psi / 2$ and $\theta=\pi / 2+\psi / 2$ (where $\psi$ runs from 0 to $4 \pi$ to complete the circle). Here $n^{+}$and $n^{-}$each consist of one annulus running twice around the solid torus. Again the generator, $\alpha$, of $H_{1}\left(n^{+}, \tau\right)$ is not in the image of $H_{2}(N, n)$ (even though $2 \alpha$ is-use integer coefficients) and so $H^{1}\left(a^{+}\right)$is nontrivial. This time the dual, $\alpha^{\prime} \in H^{1}\left(n^{+}\right)$, of $\alpha$ is not in the image of $H^{1}(N)$ however $2 \alpha^{\prime}$ is, and since $\alpha^{\prime}$ goes nontrivially to $a^{+}, 2 \alpha^{\prime}$ must also $\left(H^{1}\left(a^{+}\right)\right.$ must be torsion free in this case). Thus the generator of $H^{1}(N)$ goes again nontrivially to $H^{1}(I)$ and I runs around the solid torus.

3.3 ExAmple. A somewhat different phenomenon is illustrated by letting the tangency set consist of the two circles $\psi=0$ and $\psi=\pi$. Again $n^{+}$and $n^{-}$each consists of an annulus, but the maps from $H^{1}(N) \rightarrow H^{1}\left(n^{ \pm}\right)$are trivial. Still the generator of $H_{1}\left(n^{+}, \tau\right)$ is not in the range of $H_{2}(N, n)$ so that $H_{1}\left(n^{+}, n^{+}-a^{+}\right)$ $\approx H^{1}\left(a^{+}\right)$is not trivial and in particular: the map $H^{1}\left(n^{+}\right) \rightarrow H^{1}\left(a^{+}\right)$is injectivegeometrically, $a^{+}$and $a^{-}$contain "Čech circles" which do not bound in $n^{+}$and $n^{-}$ respectively (see Figure 3).

In this case $H^{1}(I)$ may or may not be trivial; Figure 3a shows a situation where $I$ consists of the center line of the solid torus $(r=0)$, together with two degenerate rest points, while in Figure $3 \mathrm{~b} I$ consists solely of two hyperbolic critical points which lie on the center line. The same critical points appear in $3 \mathrm{a}$, but in that example the one dimensional unstable manifold of one connects to the one dimensional stable manifold of the other. Taking this unstable situation into account as well as the degeneracy of the two other critical points in $3 \mathrm{a}$, one sees that the flow in $3 \mathrm{~b}$ can be obtained by an arbitrarily small perturbation of that in $3 \mathrm{a}$. Our theorems cannot distinguish these situations of course; however, we can prove 


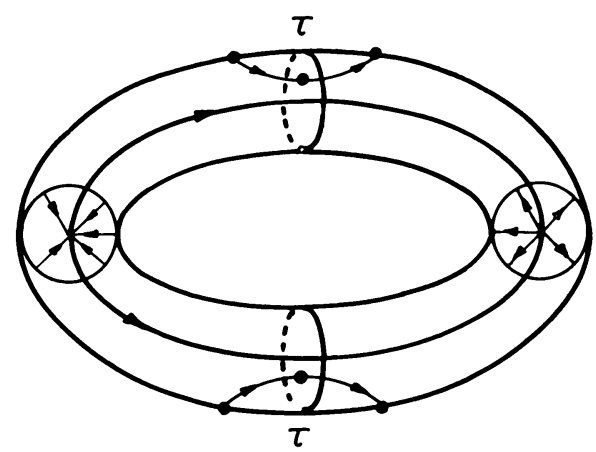

$3 a$

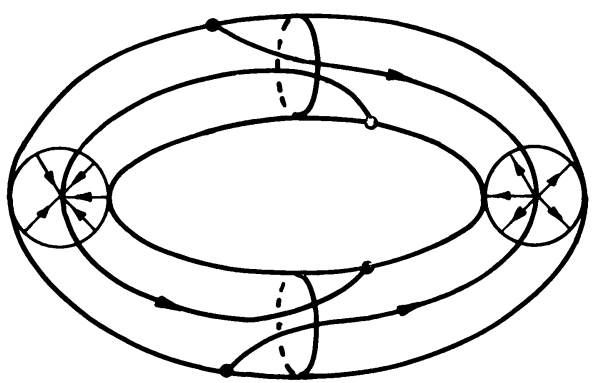

$3 b$

FIgure 3. The block $N$ is a solid torus. In 3a $I$ consists of a circle and two degenerate critical points. In $3 \mathrm{~b} I$ contains just two nondegenerate critical points. The sets $a^{+}$and $a^{-}$are the same in both pictures; each contains a circle and two points.

that if $H^{1}(I) \approx H_{1}(N, N-I) \approx 0$, then $a^{+}$cannot be connected. (Otherwise it could be-just eliminate the two degenerate critical points in 3a.) (The example is as given to illustrate the necessity of the hypothesis in 2.12 that $\varphi$ be known.) To see the statement, consider the two sequences:

$$
\begin{aligned}
\longrightarrow H_{2}\left(n^{+}, \tau\right) \longrightarrow & H_{2}\left(n^{+}, n^{+}-a^{+}\right) \\
& \stackrel{\partial}{\longrightarrow} H_{1}\left(n^{+}-a^{+}, \tau\right) \longrightarrow H_{1}\left(n^{+}, \tau\right) \longrightarrow H_{1}\left(n^{+}, n^{+}-a^{+}\right) \\
& \stackrel{\partial}{\longrightarrow} H_{2}(N-I, n) \longrightarrow H_{2}(N, n) \longrightarrow H_{2}(N, N-I) \longrightarrow .
\end{aligned}
$$

If $a^{+}$is connected, the first map in the top row is surjective; in any case, the last is injective (as we have shown already). Hence, $H_{1}\left(n^{+}-a^{+}, \tau\right) \approx H_{2}(N-I, n) \approx 0$. Then since $H_{2}(N, n)$ is not trivial and injects to $H_{2}(N, N-I)$ we see that $\hat{H}^{3-2}(I) \neq 0$.

Theorem 2.11 might also be applied here: $d_{\psi}$ represents a cocycle in $H^{1}\left(N, n^{-}\right)$, say, and $d r \wedge d \theta$ a cocycle in $H^{2}\left(N, n^{+}\right)$; their cup product is represented by $d r \wedge d \theta \wedge d \psi$ and so is the generator of $H^{3}(N, n)$.

B. Hyperbolic situations.

3.4 EXAMPLE. Not all isolated critical points are isolated as invariant sets, the elementary ones are however (among others). The typical situation might be described by the equations:

$$
\dot{x}=-x, \quad \dot{y}=+y,
$$

where $x$ and $y$ are respectively $p$ - and $q$-dimensional (real) vectors.

For $N$ it is convenient (solely for our description below) to take the set of points in $p+q$ space where $\|x\| \leqq 1$ and $\|y\| \leqq 1$. We will neglect the easily circumvented problem that $n$ is only piecewise smooth. If we let $B^{p}=\{\|x\| \leqq 1\} \subset p$-dim space and $B^{q}=\{\|y\| \leqq 1\} \subset q$-dim space, we easily check that $n^{+}=B^{p} \times \dot{B}^{q}, n^{-}=B^{q} \times \dot{B}^{p}$ and 
$\tau=\dot{B}^{p} \times \dot{B}^{q}$. Furthermore orbits through $\tau$ leave $N=B^{p} \times B^{q}$ in both directions so (except for the technicality) $N$ is an isolating block. Furthermore

$$
(N, n)=\left(B^{p}, \dot{B}^{p}\right) \times\left(B^{q}, \dot{B}^{q}\right) ;
$$

thus if $\gamma$ generates $H^{p+q}(N, n)$, and $\alpha^{\prime}$ and $\beta^{\prime}$ (resp.) generate $H^{p}\left(B^{p}, \dot{B}^{p}\right)$ and $H^{q}\left(B^{q}, \dot{B}^{q}\right)$ then $\gamma=\alpha^{\prime} \times \beta^{\prime}$. Also under the homomorphisms induced by the projections, $\alpha^{\prime}$ and $\beta^{\prime}$ go respectively to the generators $\alpha$ and $\beta$ of $H^{p}\left(N, n^{-}\right)$and $H^{q}\left(N, n^{+}\right)$so that $\gamma=\alpha \cup \beta$. Thus $H^{p+q}(N, n)$ injects to $H^{p+q}(N, N-I)$ and $\hat{H}^{\circ}(N)$ injects to $\hat{H}^{\circ}(I)(2.11)$.

Theorem 2.9 also applies and we conclude that $H_{p}\left(n^{+}, \tau\right)$ and $H_{q}\left(n^{-}, \tau\right)$ inject to $H_{p}\left(n^{+}, n^{+}-a^{+}\right)$and $H_{q}\left(n^{-}, n^{-}-a^{-}\right)$; or using duality the groups $H^{q-1}\left(n^{+}\right)$ and $\mathrm{H}^{p-1}\left(\mathrm{n}^{-}\right)$inject to $\mathrm{H}^{q-1}\left(\mathrm{a}^{+}\right)$and $\mathrm{H}^{p-1}\left(\mathrm{a}^{-}\right)$respectively. In other words, I is not empty and the asymptotic sets include what one expects (maybe more of course).

3.5 ExAmple. The previous example is a little more interesting if one takes the cross product with a compact manifold $m$ (without boundary). Then we can represent:

$$
\begin{aligned}
& N=B^{p} \times B^{q} \times m, n=\left(B^{p} \times B^{q}\right) \cdot \times m, \\
& n^{+}=B^{p} \times \dot{B}^{q} \times m, n^{-}=\dot{B}^{p} \times B^{q} \times m, \\
& \tau=\dot{B}^{p} \times \dot{B}^{q} \times m .
\end{aligned}
$$

With $\alpha, \beta$ and $\gamma$ as in 2.16, we can represent:

$$
\begin{aligned}
& H^{*}(N, n) \text { as } \gamma \otimes H^{*}(m), \\
& H^{*}\left(N, n^{+}\right) \text {as } \alpha \otimes H^{*}(m),
\end{aligned}
$$$$
H^{*}\left(N, n^{-}\right) \text {as } \beta \otimes H^{*}(m) \text {. }
$$

Then each element in $H^{*}(N, n)$ is a cup product-schematically $\alpha \otimes H^{*}(m)$ $\cup \beta \otimes H^{*}(m)=\gamma \otimes H^{*}(m)$. Theorem 2.11 then applies and shows with a little additional argument that $H^{*}(N)$ injects to $H^{*}(I)$-thus: No matter how the flow is filled in, the cohomology of $m$ is represented in $I$.

3.6 EXAMPLE. The above situation occurs naturally in Hamiltonian systems of differential equations. Let $x$ and $y$ be (real) $p$-vectors, and $z$ be a complex $q$-vector and let $H(x, y, z, \bar{z})=\sum x_{i} y_{i}+\sum z_{j} \bar{z}_{j}$; the equations are to be given by:

$$
\dot{x}=x, \quad \dot{y}=-y, \quad \dot{z}=i z .
$$

In the integral surface $H=1$, the $2 q-1$ sphere $|z|=1$ is invariant and plays the role of $m$. The isolating block $N$ is homeomorphic to $B^{p} \times B^{p} \times S^{2 q-1}$. The previous example can be applied to show a similar invariant set lies in the energy surface under small perturbations of the Hamiltonian function. A somewhat different method for this situation is given in [7]. Better theorems are available for this problem [11], [12], [13].

C. Critical points which are isolated as invariant sets.

3.7 Example. Assume that some critical point of a flow on a $d$-dimensional manifold is isolated as an invariant set and that the isolating block is a ball. 
From the sequence for the triple $\left(N, n, n^{+}\right)$(with the appropriate excision, 2.8) we see that the inclusion $\left(n^{+}, \tau\right) \rightarrow\left(N, n^{-}\right)$induces homology isomorphisms in all but dimension $d-1$; furthermore the homeomorphism

$$
H_{d-1}\left(n^{+}, \tau\right) \rightarrow H_{d-1}\left(N, n^{-}\right)
$$

is surjective with kernel generated by the fundamental cycle of $\left(n^{+}, \tau\right)$. It follows that the inclusion induced homomorphism $H_{*}\left(n^{+}, \tau\right) \rightarrow H_{*}\left(n^{+}, n^{+}-a^{+}\right)$is injective except for the possibility that this fundamental cycle goes to zero.

If $I$ is not empty (we have assumed a critical point, but want also to consider nearby flows) then $H_{d}(N-I, n) \approx H_{d-1}\left(n^{+}-a^{+}, \tau\right) \approx 0$ so that $H_{*}\left(n^{+}, \tau\right) \rightarrow$ $H_{*}\left(n^{+}, n^{+}-a^{+}\right)$must be injective with no qualification.

Finally if $I$ consists only of the critical point (the unperturbed situation again) then $H_{*}(N, N-I) \stackrel{\partial}{\approx} H_{*}\left(n^{+}-a^{+}, \tau\right)=0$ and so $H_{*}\left(n^{+}, \tau\right) \approx H_{*}\left(n^{+}, n^{+}-a^{+}\right)$and $H^{*}\left(a^{+}\right) \approx H^{*}\left(n^{+}\right)$- the cohomology of the asymptotic set and the way it sits in $n^{+}$as described by the inclusion induced cohomology map is thus determined. (However one may imagine $n^{+}$to be a cube with a knotted hole-it becomes more clear that the cohomology information is weak.)

Consider for example a case where the index of the critical point is zero. Then nearby flows may have no critical points (in fact there must be nearby flows which do not) but if $H_{*}\left(N, n^{+}\right)$is not trivial (hence $\left.I \neq \varnothing\right)$ then $H_{*}\left(n^{+}, \tau\right)$ must still inject to $H_{*}\left(n^{+}, n^{+}-a^{+}\right)$under perturbation.

Such a situation can occur (index zero, and $H_{*}\left(N, n^{+}\right) \neq 0$ ) as we can see from the following formula for the index of the field on $n$. Fix an orientation of $n$ and (the coherent orientation of) $n^{+}$. Let $p \in n^{-}$and replace $n$ by the (Euclidean) space $n-\{p\}$. Let $\tau$ be considered as the chain $\partial n^{+}$. Then the index is $1-g_{\tau}$ where $g_{\tau}$ is the degree of the Gauss map of $\tau$-namely each point of $\tau$ is mapped to its unit outward (from $n^{+}$) normal considered as a point of the unit sphere in Euclidean space. In particular, if $d$ (hence $d-2=\operatorname{dim} \tau$ ) is even, $g_{\tau}=\frac{1}{2} \mathscr{X}_{\tau}$ where $\mathscr{X}_{\tau}$ is the Euler characteristic of $\tau$. The above formula reduces to Poincaré's formula for the index of a vector field on a circle in two dimensions. In particular, the above formula (or Poincaré's) implies that any critical point in two dimensions which is isolated as an invariant set must have index less than or equal to one. This is not true in dimensions greater than two as is shown by easily constructed examples. (It is true again in even dimensions if $\tau$ is composed of spheres-but not in odd dimensions.)

An example in three dimensional space of a critical point isolated as an invariant set and with index zero is drawn in Figure 4. Next to it is a nearby flow which is nonsingular - the critical point has mutated to a periodic orbit. The first figure is to be rotated about the vertical line of asymptotic orbits; the second, in which this vertical line changes to a cylinder, is to be rotated about the dotted line (the center line of the cylinder). It is interesting that the second situation is the "stable" one, our theorems give no indication of this. 

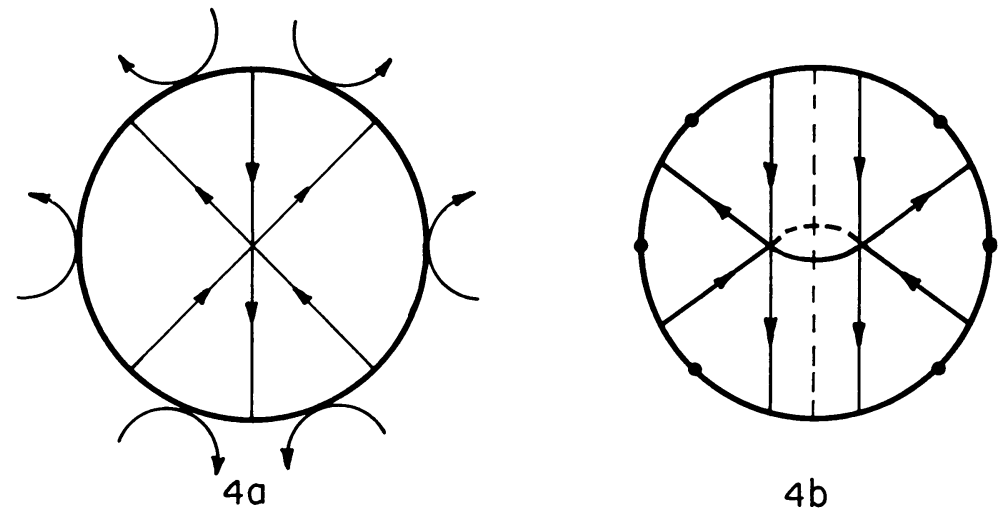

FIGURE 4

Other situations (which arise more naturally) wherein isolating blocks give some preliminary information include degenerate critical points of Hamiltonian systems [2], [10] and systems where the Hamiltonian is of the form $(y, y)-V(x)$ with $V$ a polynomial. (The isolating blocks are generally found near infinity where the highest order term dominates-the conclusions concern the set of bounded orbits. Combined with more analytical methods one might hope to get reasonably interesting results.)

4. An alternate description of isolating blocks; proof of 1.6. The results of this section will be used to prove 1.5 and 1.6; the latter proof is sketched in this section the former given in detail in the next.

A. Local surfaces of section.

4.1 Definition. A local surface of section, $S$, for $\varphi_{V}$ will mean a smooth open submanifold of $M$ with codimension one and such that $V$ is never tangent to $S$.

Thus, if $N$ is an isolating block, the interiors of $n^{+}$and $n^{-}$are local surfaces of section (1.3 and 1.4). We omit the proof of the following easy corollary:

4.2 Corollary. Let $S$ be a local surface of section for $\varphi_{V}$ and let $S^{\prime} \subset S$ be an open submanifold of $S$ with the same dimension and with compact closure in $S$.

Then there is an $\varepsilon>0$ and a neighborhood $\nu$ of $V$ in the $C^{0}$ topology such that $S^{\prime}$ is a local surface section for all $W \in \nu$, and furthermore the mapping

$$
j_{w}: S^{\prime} \times(-\varepsilon, \varepsilon) \rightarrow M
$$

defined by $j_{W}(p, t)=\varphi_{W}(p, t)$ is a homeomorphism for all (smooth) $W \in \nu$.

4.3 Definition (CF. 2.3). Let $S_{+}$and $S_{-}$be disjoint local surfaces of section for for $\varphi_{V}$ and define $\sigma$ from $S_{+}$to the extended reals by

$$
\sigma(p)=\sup \left\{t \in R^{+} \mid p \cdot[0, t] \cap S_{-}=\varnothing\right\} .
$$

Also, let $D$ (or $D(V)$ if more vector fields are being considered) be that subset of $S_{+}$such that $p \cdot \sigma(p) \in S_{-}$. (In particular, $\sigma(p)$ is finite; however this is not equivalent.) Further, let $D_{c}(V)$ be the subset of $D(V)$ where $\sigma$ is continuous. 
Finally let $\pi_{V}: D(V) \rightarrow S_{-}$be given by $\pi_{V}(p)=p \cdot \sigma(p)$.

4.4 Corollary. $D$ and $D_{c}$ are open and $\sigma$ is upper semicontinuous on $D$; also $D-D_{c}$ consists of exactly those points $p$ of $D$ for which there is a $t$ such that $0 \leqq t<\sigma(p)$ and $p \cdot t \in \mathrm{Cl}\left(S_{-}\right)-S_{-}$.

The proof that $D$ is open and $\sigma$ is upper semicontinuous is similar to that sketched in 2.6. (Here, one might use continuity in initial conditions with 4.2 to see that if $p$ is carried to $S_{-}$then a neighborhood, in $S_{+}$or in $M$, of $p$ is also.)

If $p$ is a point of discontinuity of $\sigma$ then there is a sequence $p_{n} \rightarrow p$ for which $\lim \sigma\left(p_{n}\right) \neq \sigma(p)$ hence $\lim \sigma\left(p_{n}\right)<\sigma(p)$. If the limit is $t$, then (compactness) $p \cdot t$ must be in $\mathrm{Cl}\left(S_{-}\right)$but not in $S_{-}$. On the other hand, if $p \cdot t \in \mathrm{Cl}\left(S_{-}\right)$with $t<\sigma(p)<\infty$, there must be points close to $p \cdot t$ in $S_{-}$; hence (using $S_{+}$as a local surface of section and in particular 4.2) points in $S_{+}$near $p$ which meet $S_{-}$in times close to $t$. It follows that $p$ must be a point of discontinuity of $\sigma$. That $D_{c}$ is open now follows: if an orbit segment from $U$ to $S_{-}$misses $\mathrm{Cl}\left(S_{-}\right)-S_{-}$(closed since $S_{-}$is an open submanifold) then nearby orbit segments must also.

4.5 Lemma. Let $S_{+}$and $S_{-}$be disjoint local surfaces of section for $\varphi_{V}$ and let $F$ be a compact set in $D_{c}(V)$. Let $S_{+}^{\prime}$ and $S_{-}^{\prime}$ be local surfaces of sections whose closures are contained in $S_{+}$and $S_{-}$respectively, and such that $F \subset S_{+}^{\prime}$ and $\pi_{V}(F) \subset S_{-}^{\prime}$.

Then there is a neighborhood $\nu$ of $V$ in the $C^{0}$ topology such that for (smooth) $W \in \nu, S_{+}^{\prime}$ and $S_{-}^{\prime}$ are local surfaces of section and $F \subset D_{c}(W)$.

By $4.2 S_{+}^{\prime}$ and $S_{-}^{\prime}$ are local surfaces of section of $w$ if $\nu$ is small enough. The main point of the proof is that given $T(>0), W$ close to $V$ implies $\varphi_{W}(p, t)$ close to $\varphi_{V}(p, t)$ for $-T \leqq t \leqq T$. Choosing $T$ bigger than the upper bound of $\sigma$ on $F$, one sees (4.2 again) that (for small enough $\nu$ ) $p$ is carried by $W$ to $S_{-}^{\prime}$ in time close to $\sigma_{v}(p)$ and that the orbit segment cannot meet $\mathrm{Cl}\left(S_{-}^{\prime}\right)-S_{-}^{\prime}$.

4.6 Corollary. Let $\operatorname{Seg}\left(F, \pi_{V}(F)\right)$ denote the union of the orbit segments $\varphi(p,[0, \sigma(p)])$, where $p \in F$.

If $\operatorname{Seg}\left(F, \pi_{V}(F)\right)$ meets $F$ only in initial points of the orbit segments then $\pi_{V} \mid F$ is a homeomorphism and $\operatorname{Seg}\left(F, \pi_{V}(F)\right) \approx F \times J$ where $J$ denotes the unit interval.

The corresponding statement holds in some $C^{0}$ neighborhood of $V$ (for smooth flows).

Proof. The hypotheses imply that $\pi_{V}$ is one-one, and (since $\sigma$ is continuous) the inverse of $\pi_{V}$ is continuous. Also the perturbed orbit segments must again miss $F$ (excepting initial points) if $W$ is close enough to $V$.

The following lemma allows us to replace the notion of an isolating block with a technically more complicated one which is a little easier to work with in the proofs: 
4.7 Lemma. Let $S_{+}$and $S_{-}$be disjoint local surfaces of section and let $\tilde{n}^{+} \subset S_{+}$ be a compact submanifold with the same dimension as $S_{+}$and with boundary $\tilde{\tau}$. Assume also that $\tilde{\tau} \subset D_{c}$, that $T=\operatorname{Seg}(\tilde{\tau}, \pi(\tilde{\tau})) \approx \tilde{\tau} \times J$ and that $\pi(\tilde{\tau})$ bounds a compact submanifold $\tilde{n}^{-} \subset S_{-}$.

Finally, assume that $\tilde{n}=\tilde{n}^{+} \cup T \cup \tilde{n}^{-}$is a (piecewise smooth) manifold which bounds (in M) a submanifold $\tilde{N}$. (That $\tilde{n}$ is a piecewise smooth manifold implies $\operatorname{Seg}(\tilde{\tau}, \pi(\tilde{\tau}))$ meets $\tilde{n}^{+}$only in $\tilde{\tau}$.) And that $\tilde{n}^{+}$(resp. $\left.\tilde{n}^{-}\right)$constitutes the set of incoming (resp. outgoing) points of $\tilde{N}$.

Then there is an isolating block $N$ containing $\tilde{N}$ with the property that $\tilde{n}^{+} \subset n^{+}$ and $\tilde{n}^{-} \subset n^{-}$. Furthermore, $\tau$ is a deformation of $T$, and $\tilde{n}^{+}, \tilde{n}^{-}$and $\tilde{N}$ are strong deformation retractions of $N$.

In particular, defining asymptotic and invariant sets of $\tilde{N}$ as in Definition 2.1, these sets are the same as those for $N$.

To construct $N$ one uses a collar $C$ of the boundary $\tilde{\tau}$ of $\tilde{n}^{+}$which is outside of $n^{+}$and contained in $D_{c} ; N$ is obtained by "rounding off the corners" as indicated in Figure 5.

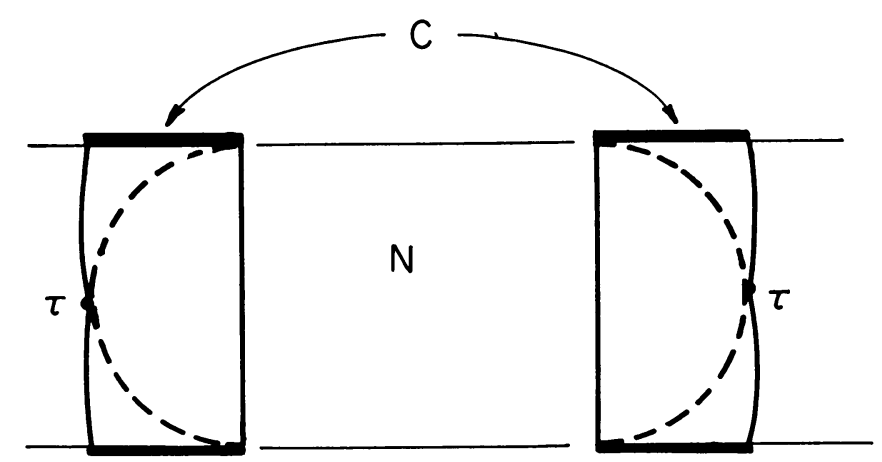

FIGURE 5

B. Proof of Theorem 1.6. To prove 1.6, select $\tilde{n}^{+} \subset n^{+}$so that $\partial \tilde{n}^{+} \cap a^{+}=\varnothing$ and $\mathrm{Cl}\left(n^{+}-\tilde{n}^{+}\right)$is a collar on $n^{+}$which does not meet $a^{+}$. Then $\partial \tilde{n}^{+}$satisfies the conditions of 4.5 and 4.6 with $S_{+}$and $S_{-}$the interiors of $n^{+}$and $n^{-}$respectively. These are applied with $S_{+}^{\prime}$ and $S_{-}^{\prime}$ being neighborhoods of $\tilde{n}^{+}$and $\tilde{n}^{-}$obtained by adding that half of the relevant collar adjacent to $\tilde{n}^{+}$or $\tilde{n}^{-}$.

Now the conditions of 4.7 are satisfied by $\tilde{n}^{+}$and $\partial \tilde{n}^{+}=\tilde{\tau}$ for $V$ and it is clear how to show they are satisfied for nearby flows. The proof is completed by applying 4.7.

5. The construction of an isolating block for an isolated invariant set. Given an isolated invariant set $I$ and an isolating neighborhood $U$, our aim is to construct an isolating block for $I$ which lies in $U(1.1,1.2,1.3,1.4)$. We do this by first constructing local surfaces of section for the asymptotic orbits in $U(5.1)$ and then applying Lemma 4.7. 
In A a neighborhood of the asymptotic orbits (not in $I$ ) is constructed and a quotient space (identifying orbit components to points) is defined. In B the quotient space is shown to be a (Hausdorff) manifold. In $\mathrm{C}$ the neighborhood is shown to be a fibre bundle over the quotient space with fibre homeomorphic to the real line. This provides sections which are somewhat modified in D. In E Lemma 4.7 is shown to apply and the proof is completed.

A. A neighborhood, $E$, of the asymptotic orbits. With $I$ and $U$ as above:

5.1 Definition. Let $A^{+} \equiv\left\{p \mid p \cdot R^{+} \subset \bar{U}\right\}, A^{-} \equiv\left\{p \mid p \cdot R^{-} \subset \bar{U}\right\}, \quad A_{t} \equiv A^{+} \cdot t \cup$ $A^{-} \cdot(-t)$.

Note that $A^{+}$and $A^{-}$are compact sets with intersection $I$, and that the sets $A_{t}$ are compact and decrease with increasing $t$.

5.2 Lemma. Given an open set $W \supset I$, there exists a real number $\rho(W)>0$ such that for all $t>\rho(W), A_{t} \subset W$.

Proof. $\bigcap_{t>0} A_{t}$ is an invariant set in $U$, hence in $I$ (it must equal $I$ in fact). The lemma follows from the compactness of $M$ and $I$.

5.3 Lemma. Let $W$ be an open set about I with $\mathrm{Cl}(W) \subset U$ and let $\rho=\rho(W)$ (5.2). Then for each $p \in \mathrm{Cl}(U) \cap A^{+}-\mathrm{Cl}(W)$ there is a local surface of section (4.1) $D_{p}$ containing $p$ and satisfying:

(1) $\mathrm{Cl}\left(D_{p}\right) \cap W=\varnothing$.

(2) $\mathrm{Cl}\left(D_{p}\right) \times[0,3 \rho]$ is homeomorphic to $\mathrm{Cl}\left(D_{p}\right) \cdot[0,3 \rho]$ under the map $(p, t)$ $\rightarrow p \cdot t$.

(3) $\mathrm{Cl}\left(D_{p}\right) \cdot[\rho, 3 \rho] \subset W$.

Proof. Properties (1), (2) and (3) hold if $\mathrm{Cl}\left(D_{p}\right)$ is replaced by $p$; by "continuity in initial conditions" such a $D_{p}$ can be found.

5.4 Definition. (1) Let $E_{p} \equiv D_{p} \cdot(0, \rho)$. (Observe $E_{p}$ is open in M.)

(2) Let $E=\bigcup E_{p}$ over $p \in \mathrm{Cl}(U) \cap A^{+}-\mathrm{Cl}(W)$. Also let $D \equiv \bigcup D_{p}$ over $p \in \mathrm{Cl}(U) \cap A^{+}-\mathrm{Cl}(W)$. Note that $D \subset M-\mathrm{Cl}(W)$.

(3) Define an equivalence relation, $\sim$, on $E$ as follows: $p \sim q$ if and only if there is an orbit segment $\operatorname{Seg}(p q)$ connecting $p$ and $q$ and $\operatorname{Seg}(p q) \subset E .(\sim$ is clearly an equivalence relation.)

(4) Let $B \equiv E / \sim$ and let $\pi: E \rightarrow B$ be the quotient map.

$B$. The base space $B$. The main portion of the proof consists of showing $E$ is a fibre bundle over $B$ and admits a section-the latter being made essentially by pasting all the $D_{p}$ 's together.

5.5 LemMA. Let $p$ and $q$ be in $E$ and let Seg $(p q)$ be an orbit segment (not necessarily in $E$ ) which connects $p$ and $q$. Let $|p q|>0$ be the positive real number such that either $p \cdot|p q|=q$ or $q \cdot|p q|=p$ (the "length" of $\operatorname{Seg}(p q)$ ).

Then $\operatorname{Seg}(p q) \subset E$ if and only if $|p q|<2 \rho$.

Proof. We first show that if $p^{\prime}$ and $q^{\prime}$ are in $D$ then either (a) $\left|p^{\prime} q^{\prime}\right|>3 \rho$ or (b) $\left|p^{\prime} q^{\prime}\right|<\rho$. 
If this were not true there would be a $t^{\prime}$ between $\rho$ and $3 \rho$ with (say) $p^{\prime} \cdot t^{\prime}=q^{\prime}$. But $p^{\prime} \cdot t^{\prime}$ would then be in $W(5.3,3)$ whereas $q^{\prime} \in D$ is not.

Now suppose $\operatorname{Seg}(p q) \subset E$ and $|p q| \geqq 2 \rho$ and let $p \cdot|p q|=q$. Since $\operatorname{Seg}(p q) \subset E$, $r=p \cdot 2 \rho \in E$. This implies the existence of $r^{\prime} \in D$ and $t^{\prime}<\rho$ such that $r=r^{\prime} \cdot t^{\prime}$ (5.4, (1) and (2)). Also, $p=p^{\prime} \cdot t^{\prime \prime}$ with $p^{\prime} \in D$ and positive $t^{\prime \prime}<\rho$. Thus $r^{\prime}=p^{\prime}$ $\cdot\left(t^{\prime \prime}+2 \rho-t^{\prime}\right)$. But $\rho<t^{\prime \prime}+2 \rho-t^{\prime}<3 \rho$ and this contradicts the fact that $p^{\prime}$ and $r^{\prime}$ are in $D$. Thus $|p q|>2 \rho$ implies $\operatorname{Seg}(p q) \notin E$.

Suppose then that $|p q|<2 \rho$. Since $p$ and $q$ are in $E$ by hypothesis, there exist $p^{\prime}, q^{\prime} \in D$ and positive $t^{\prime}, t^{\prime \prime}<\rho$ such that $p=p^{\prime} \cdot t^{\prime}$ and $q=q^{\prime} \cdot t^{\prime \prime}$. Then

$$
p^{\prime} \cdot\left(t^{\prime}+|p q|-t^{\prime \prime}\right)=q^{\prime}
$$

Since $p^{\prime}$ and $q^{\prime}$ are in $D$, and $t^{\prime}+|p q|-t^{\prime \prime}<3 \rho$, it must be that $t^{\prime}+|p q|-t^{\prime \prime}<\rho$. It follows that $\operatorname{Seg}\left(p^{\prime} q^{\prime}\right)$ and $\operatorname{Seg}\left(q^{\prime} q\right)$ hence $\operatorname{Seg}\left(p^{\prime} q\right)$ lie in $E$ and finally, since the last contains $p$, that $\operatorname{Seg}(p q) \subset E$.

5.6 Corollary. For each $x \in B, \pi^{-1}(x)$ is an open arc whose initial point lies in $M-W$. Also each maximal half orbit in $A^{+}-I$ meets $E$ in exactly one open interval.

Proof. $\pi^{-1}(x)$ is either an arc or a circle. If it were a circle there would be arbitrarily long orbit segments in $E$ contradicting 5.5 . The initial point must be in $M-W$ since it is a limit of points in $D \subset M-\bar{W}$ (by 5.4). Also, every orbit in $A^{+}-I$ meets $E$ in a nonvoid collection of intervals; but there can be at most one of these since each must have length at least $\rho$. By choice of $\rho(5.3)$ there is at most one such interval (the remainder of the orbit lies in $W$ ).

\subsection{LeMMA. The projection $\pi$ is open as well as continuous.}

That $\pi$ is continuous follows from the definition of the quotient topologynamely $\nu \subset B$ is open if $\pi^{-1}(\nu)$ is open. To see that $\pi$ is open, let $\nu \subset E$ be open and consider $\pi^{-1}(\pi(\nu))$. Now $p$ is in this set if and only if there is a $q \in \nu$ and an orbit segment $\operatorname{Seg}(p q)$ such that $\operatorname{Seg}(p q) \subset E$. But if this is the case for $p$, then by openness of $E$ and $\nu$ and by 5.5 it is true for points in a neighborhood of $p$, thus $\pi^{-1}(\pi(\nu))$ is open and so $\pi(\nu)$ is open.

5.8 LEMMA. $B$ admits the structure of a smooth Hausdorff manifold in such a way that $\pi$ is differentiable ( $E$ has the smooth structure inherited from $M$ ).

Proof. Let $D_{p}$ be any of the local sections of 5.3 and define $f_{p}: D_{p} \rightarrow B$ by $f_{p}(q)=\pi(q \cdot(0, \rho))$.

To see $f_{p}$ is continuous, let $V \subset B$ be open. Then $\pi^{-1}(V)$ is open in $E$ so meets $D_{p} \cdot(0, \rho)$ in an open set. Projecting this into $D_{p}$ by the flow one obtains the inverse image of $V$ under $f_{p}$ which is then open.

Similarly $f_{p}$ is open, for if $V \subset D_{p}$ is open, then $V \cdot(0, \rho)$ is open in $E$ and so $\pi(V \cdot(0, \rho))=f_{p}(V)$ is open in $B$. 
Also $f_{p}$ is one-one: If $f_{p}(q)=f_{p}\left(q^{\prime}\right)$, then there is an (open) orbit segment Seg $\left(q q^{\prime}\right)$ in $E$. But this orbit segment is no longer than $2 \rho(5.5)$, and since no point of $D_{p}$ returns in time less than $3 \rho(5.3,2) q$ and $q^{\prime}$ must be the same point.

The functions $f_{p}$ thus define coordinate patches on $B$ so that $B$ is a manifold. Furthermore the overlap maps, $f_{p}^{-1} \circ f_{p^{\prime}}$ are just the maps between the sections defined by the flow and so are smooth. Similarly $\pi$ is smooth.

It remains to prove that $B$ is Hausdorff: Suppose $x$ and $y$ are distinct points of $B$ and that every neighborhood of $x$ meets every neighborhood of $y$. Now $\pi^{-1}(x)$ and $\pi^{-1}(y)$ are disjoint in $E$; but even their closures (in $M$ ) must be: otherwise they would lie in the same orbit, and share an endpoint. Using 5.5, this endpoint could be in $E$ and this is not possible with $\pi^{-1}(x) \neq \pi^{-1}(y)(5.4,(3))$. Now suppose $\nu_{x}$ and $\nu_{y}$ are neighborhoods (in $E$ ) of $\pi^{-1}(x)$ and $\pi^{-1}(y)$ respectively. Since $\pi\left(\nu_{x}\right)$ meets $\pi\left(\nu_{y}\right)$ there is an orbit segment in $E$ (lergth $<2 \rho$ ) connecting $\nu_{x}$ to $\nu_{y}$. On letting $\nu_{x}$ and $\nu_{y}$ collapse to $\pi^{-1}(x)$ and $\pi^{-1}(y)$ we see that these latter must lie on the same orbit segment. Now an orbit segment passing near points in both of these segments must have points near the initial point of one of them; then either it has points near the initial point of the other, or its initial point is near the other. In either case, since initial points are in $U-W(5.6)$, there is an orbit segment in $E$ with length $\leqq \rho$ passing close to $\pi^{-1}(x)$ and $\pi^{-1}(y)$. By $5.5, \pi^{-1}(x)$ and $\pi^{-1}(y)$ lie in the same orbit segment in $E$ contradicting the assumption $\pi^{-1}(x) \neq \pi^{-1}(y)$.

C. The bundle space $E$.

5.9 Lemma. Define functions $\sigma^{+}$and $\sigma^{-}$on $E$ by

$$
\sigma^{+}(p)=\sup \{t \mid p \cdot t \in E\}, \quad \sigma^{-}(p)=\inf \{t \mid p \cdot t \in E\} .
$$

Then $\sigma^{+}$is lower semicontinuous and $\sigma^{-}$is upper semicontinuous.

Given $\varepsilon>0$ there is a point $r \in D$ on the orbit segment $p \cdot\left(\sigma^{-}(p), \sigma^{-}(p)+\varepsilon\right)$. (This segment is contained in $E$ if $\varepsilon$ is small.) Let $D_{q}$ be the local section of 5.3 which contains $r$. Then if $p^{\prime}$ is close to $p$, the orbit segment through $p^{\prime}$ must meet $D_{q}$. Also the orbit segment from $D_{q}$ to $p^{\prime}$ must be shorter than $2 \rho$ so (5.5) in $E$. Thus $\sigma^{-}\left(p^{\prime}\right) \leqq \sigma^{-}(p)+\varepsilon$ and $\sigma^{-}$is upper semicontinuous.

Given $\varepsilon>0$ and $p \in E$ there is a point $r$ of $D$ such that $q \equiv r \cdot(\rho-\varepsilon / 2)$ lies on the orbit segment $p \cdot\left(\sigma^{+}(p)-\varepsilon, \sigma^{+}(p)\right)$ (also in $E$ for small $\varepsilon>0$ ).

Now let $p^{\prime}$ be close to $p$. Then there is a point $q^{\prime}$ on the same orbit as $p^{\prime}$ and close to $q$. Since $p q$ has length less than $2 \rho$ so will $\operatorname{Seg}\left(p^{\prime} q^{\prime}\right)$. Also, since $\operatorname{Seg}(p q)$ meets the section of $D$ containing $r$, so will $\operatorname{Seg}\left(p^{\prime} q^{\prime}\right)$ implying in particular that $q^{\prime} \in E$ $(5.4,1)$. It follows that $\operatorname{Seg}\left(p^{\prime} q^{\prime}\right) \subset E(5.5)$ and finally $\lim \inf \sigma^{+}\left(p^{\prime}\right) \geqq \sigma^{+}\left(p^{\prime}\right)$ as $p^{\prime} \rightarrow p$.

5.10 LEMMA. $E$ admits the structure of a smooth fiber bundle over $B$ with fiber homeomorphic to the real line. In particular $E$ admits a (smooth) section $S$.

Furthermore $S$ can be taken so that:

(1) $S$ is never tangent to the fiber and so is a local surface of section for the flow. 
(2) $S \subset W$.

(3) Each half orbit in $A^{+}-I$ meets $S$ in exactly one point.

(4) $I \cap \mathrm{Cl}(S)=\varnothing$.

Proof. We can assume that the local sections, $D_{p}$, are smooth images of a standard $d-1$ dimensional ball $\Delta$ in Euclidean $d-1$ dimensional space. Call the map $\alpha_{p}$. Then $\pi^{-1} \circ f_{p}\left(D_{p}\right)$ is diffeomorphic to the open set $\nu$ in $d$-dimensional space defined below. In the definition $\varepsilon>0$ and less than $\rho$ is introduced so that the argument, $\alpha_{p}(q) \cdot \varepsilon$, of $\sigma^{+}$and $\sigma^{-}$is in $E$ :

$$
\nu \equiv\left\{(q, t) \mid q \in \Delta \text { and } \exists \varepsilon>0 \ni \sigma^{-}\left[\alpha_{p}(q) \cdot \varepsilon\right]<t<\sigma^{+}\left[\alpha_{p}(q) \cdot \varepsilon\right]\right\} .
$$

Since $\sigma^{+}$and $\sigma^{-}$are respectively lower and upper semicontinuous (5.9) and $\sigma^{+}>\sigma^{-}, \nu$ is diffeomorphic to the product $\Delta \times R$ with a fiber preserving diffeomorphism. The composite provides the local product structure of $E$. The overlap maps are easily verified to be smooth bundle morphisms since they are essentially defined by the flow.

That $E$ admits a section follows from the theorem that a bundle over a paracompact (Hausdorff) manifold with contractible fiber admits a section. Such a section can then be approximated by a smooth one which is never tangent to the fiber. That each half orbit in $A^{+}-I$ meets $S$ follows from 5.6. That $I \cap \mathrm{Cl}(S)=\varnothing$ follows from the construction of $E$-each orbit segment in $E$ meets $D$, which lies outside of $W$, and so is disjoint from $I$; also each segment is no longer than $2 \rho$ so $\mathrm{Cl}(E) \cap I=\varnothing$. The section can be pulled into $W$ using the flow in the obvious way (and using the definition of $\rho$ in 5.3 with $5.3,3$ ).

D. Sections for $A^{+}$and $A^{-}$. The section obtained for $E$ will play the role of $S_{+}$ in 4.7. $S_{-}$is obtained in a similar way by replacing $A^{+}$by $A^{-}$. Before choosing $\tilde{n}^{+}$and $\tilde{n}^{-}$of 4.7 we need to modify these sections somewhat.

5.11 Lemma. Let $S$ be the section for $E$ obtained in 5.10. Then $A^{+} \cap S_{+}$is compact.

Proof. If $A^{+} \subset S_{+}$is not compact then the intersection must have a limit point $p$ in $\mathrm{Cl}\left(S_{+}\right)-S_{+}$. Since $p$ is in $A^{+}-I\left(A^{+}\right.$is compact and $\left.\mathrm{Cl}\left(S^{+}\right) \cap I=\varnothing(5.10)\right)$ there is a time $t \neq 0$ such that $p \cdot t \in S_{+}$(5.10). But since $S_{+}$is a local surface of section for the flow, all points close to $p$ must meet $S_{+}$in times close to $t$; in particular those in $A^{+} \cap S_{+}$which are close to $p$. This contradicts the statement that each half orbit in $A^{+}$meets $S_{+}$exactly once (5.10).

The main lemma is:

5.12 LeMma. Let $I$ be an isolated invariant set and let $U$ be an isolating neighborhood for I. Let $A^{+}$and $A^{-}$be as in 5.1 and suppose $A^{+} \neq \varnothing, A^{-} \neq \varnothing$. Let $\mathrm{Cl}(W) \subset U$ where $W$ is open and contains I. Finally let $\rho=\rho(W)$ of 5.2.

Then there exist local surfaces of section $S_{+}$and $S_{-}$in $W$ with the following properties: 
1. $\mathrm{Cl}\left(S_{+}\right) \cap \mathrm{Cl}\left(S_{-}\right)=\varnothing$.

2. $A^{+} \cap S_{+}$and $A^{-} \cap S_{-}$are compact.

3. $A^{+} \cap\left(\mathrm{Cl}\left(S_{+}\right)-S_{+}\right)=A^{-} \cap\left(\mathrm{Cl}\left(S_{-}\right)-S_{-}\right)=\varnothing$.

4. For $p \in A^{+} \cap \partial U \exists t(p)<2 \rho \ni p \cdot t(p) \in S_{+}$. If $t^{\prime}>t(p)$ then $p \cdot t^{\prime} \in W$.

5. For $p \in A^{-} \cap \partial U \exists t>-2 \rho \ni p \cdot t \in S_{-}$. If $t^{\prime}<t(p)$ then $p \cdot t^{\prime} \in W$.

6. $\mathrm{Cl}\left(S_{+}\right) \cap I=\mathrm{Cl}\left(S_{-}\right) \cap I=\varnothing$.

Proof. Let $S_{+}^{\prime}$ and $S_{-}^{\prime}$ be the sections provided by 5.10 .

Numbers 2, 4, 5, and 6 are satisfied by $S_{+}^{\prime}$ and $S_{-}^{\prime}$ in view of 5.10, 5.11 and 5.5. Also the sets $A^{+} \cap S_{+}$and $A^{-} \cap S_{-}$are disjoint compact sets-their intersection is contained in $I$ and 6 prevents its being nonempty. Subsections $S_{+}$and $S_{-}$which have disjoint compact closures in $S_{+}^{\prime}$ and $S_{-}^{\prime}$ (resp.) and which contain $A^{+} \cap S_{+}$ and $A^{-} \cap S_{-}$respectively can thus be chosen. Number 3 follows (since $A^{+} \cap S_{+}$ $\left.\subset S_{+}^{\prime}\right)$.

To choose $\tilde{n}^{+}$and $\tilde{n}^{-}$we need the following lemmas:

5.13 Definition. For $p \in U$, let $O(p)$ denote the connected component of $p \cdot R \cap \mathrm{Cl}(U)$ which contains $p \cdot O^{+}(p)$ and $O^{-}(p)$ denote respectively the positive and negative (from $p$ ) halves of $O(p)$.

5.14 Lemma. If $p_{n} \rightarrow I$ then $\lim \sup O^{+}\left(p_{n}\right) \subset A^{-}$and $\lim \sup O^{-}\left(p_{n}\right) \subset A^{+}$.

Proof. If $r \in \lim \sup O^{+}\left(p_{n}\right)$ then $\exists t_{n}>0 \ni p_{n}-t_{n} \rightarrow r$. If $t_{n}$ is bounded then $r \in I \subset A^{-}$. If $t_{n} \rightarrow \infty$, then for every fixed $t^{\prime}, p_{n} \cdot\left(t_{n}-t^{\prime}\right)$ must eventually be in $\mathrm{Cl}(U)$ which implies $r \cdot\left(-t^{\prime}\right) \in \mathrm{Cl}(U)$. Since $t^{\prime}$ is arbitrary, this implies $r \in A^{-}$. There is a similar proof for the other statement.

5.15 Lemma. Let $q_{n} \rightarrow I$ and suppose for all $n, q_{n} \notin A^{-}$. Then for sufficiently large $n, O\left(q_{n}\right)$ meets $S_{+}$exactly once.

Proof. Since $q_{n} \notin A^{-}, \exists t_{n}<0 \ni q_{n} \cdot t_{n} \in \partial U$ and $q_{n} \cdot t_{n} \in O\left(q_{n}\right)$. Let $r$ be a limit point of $q_{n} \cdot t_{n}$. Then $r \in A^{+} \cap \partial U$. Thus the orbit $O(r)$ meets $S_{+}$exactly oncein fact it meets $\mathrm{Cl}\left(S^{+}\right)$exactly once (5.12). It follows that $O\left(q_{n}\right)$ meets $S_{+}$at least once provided $q_{n} \cdot t_{n}$ is close enough to $r$. I.e. if $n$ is large enough. Now suppose $O\left(q_{n}\right)$ meets $S_{+}$(at least) twice for arbitrarily large $n$. Then if the length of the orbit segment (in $\mathrm{Cl}(U)$ ) between the intersections is bounded, $O(r)\left(\subset A^{+}\right)$ would meet $S^{+}$twice contradicting 5.12. If it were unbounded, then the midpoint of the segment would converge to $I$ and from 5.14, the later intersections would converge to a point in $A^{-}$contradicting the fact that $\mathrm{Cl}\left(S_{+}\right) \cap \mathrm{Cl}\left(S_{-}\right)=\varnothing$ (5.12).

5.16 Lemma. Let $S_{+}^{\prime}$ and $S_{-}^{\prime}$ denote sections as provided in 5.12. Then there exist subsections $S_{+}$and $S_{-}$satisfying the conditions of 5.12 and such that if $p \in S_{+} \cup S_{-}$then $O(p)$ meets each of $S_{+}$and $S_{-}$in at most one point. In particular, $p \notin A^{+} \cap S_{+} \cup A^{-} \cap S_{-}$implies $O(p)$ meets both sections exactly once and otherwise $O(p)$ meets only one section. 
Proof. Use 5.15 to find an open neighborhood $W^{\prime}$ of $I$ such that $q \in W$ implies $O(q)$ meets each of $S_{+}^{\prime}$ and $S_{-}^{\prime}$ at most once. $S_{+}$is then $O(W) \cap S_{+}^{\prime}$ and $S_{-}$ $=O(W) \cap S_{-}^{\prime}$ where $O(W)=\bigcup_{p \in W} O(p)$.

5.17 Corollary. As $W^{\prime}$ collapses to $I, S_{+}$and $S_{-}$collapse to $S_{+} \cap A^{+}$and $S_{-} \cap A^{-}$respectively.

Proof. 5.15.

E. Completion of the proof of 1.5. We are now in a position to complete the proof of Theorem 1.5 via Lemma 4.7. Before doing so we treat the special case when one of $A^{+}$or $A^{-}$is empty:

5.18 Lemma. If $A^{-}$is empty then $A^{+}-\left(A^{+} \cap \partial U\right)$ is open and is in particular an isolating neighborhood of $I$. As a consequence, $S_{+}$is a compact manifold without boundary; furthermore $S_{+}$separates $M$ and so bounds an isolating block $N$ for $I$.

Proof. Suppose $A^{+}-A^{+} \cap \partial U$ is not open. Then there is a sequence of points $p_{n} \in U$ such that $p_{n} \rightarrow A^{+}$where for all $n, p_{n} \notin A^{+}$. Now since $p_{n} \rightarrow A^{+}$, the length of $O\left(p_{n}\right)$ must tend to $\infty$. Thus the midpoint of $O\left(p_{n}\right)$ tends to $I$. By compactness of $\mathrm{Cl}(U)$, lim sup $\mathrm{O}^{+}\left(p_{n}\right)$ is nonempty, but this set is contained in $A^{-}$(5.14) contradicting the assumption that $A^{-}$is empty.

It follows that $S_{+}$is a closed manifold since no boundary points of $S_{+}$are in $A^{+}$. To see that $S_{+}$separates $M$, consider first an $\operatorname{arc} \gamma$ from $\partial U$ to $I$ meeting $I$ in exactly one point-an endpoint. Since each point, $p$, of $\gamma$ (other than the distinguished endpoint) is in $A^{+}-I$, there is a time $t(p)$ such that $p \cdot t(p) \in S_{+}$. Since $S_{+}$has no boundary, the function $t(p)$ is continuous (cf. proof of 4.4-a similar one works). Also $t(p)$ must be positive for $p \in \partial U$ and must be negative for $p$ close to $I$ (5.12). Thus there exists $p \in \gamma$ for which $t(p)=0$ so that $p \in S_{+}$. It follows that $S_{+}$separates $\partial U$ from $I$ in $U$ but since $\partial U$ separates $M, S_{+}$must separate $M$. The interior of $N$ is of course the union of the components of $M-S_{+}$which meet $I$, in this case all of them.

We can now consider the case when neither of $A^{+}$or $A^{-}$are empty. From 5.16 we see that the set $D_{c}$ associated with $S_{+}$and $S_{-}$as in 4.3 is just $S_{+}-S_{+} \cap A^{+}$, and the map $\pi: D_{c} \rightarrow S_{-}$is a homeomorphism from $S_{+}-S_{+} \cap A^{+}$to $S_{-}-S_{-}$ $\cap A^{-}$.

Select $\tilde{n}^{+} \subset S_{+}$as described in 4.7 with $\partial \tilde{n}^{+}=\tilde{\tau}$ in $D_{c}\left(=S_{+}\right)$and with $S_{+} \cap A^{+}$ $\subset \tilde{n}^{+}$. By 5.16 we see that $T=\operatorname{Seg}(\tilde{\tau}, \pi(\tilde{\tau})) \approx \tilde{\tau} \times J$.

We want now to show that $\pi(\tilde{\tau})$ separates $\partial S_{-}$from $S_{-} \cap A^{-}$and so bounds a compact manifold $\tilde{n}^{-} \supset S_{-} \cap A^{-}$: Suppose, then, that $\gamma$ is an arc from $\partial S_{-}$to $S_{-} \cap A^{-}$which meets $S_{-} \cap A^{-}$in exactly one point-an endpoint. Then the preimage of $\gamma$ under $\pi$ runs from $\partial S_{+}$to $S_{+} \cap A^{+}$and so must meet $\tilde{\tau}$. Thus $\gamma$ meets $\pi(\tilde{\tau})$ and $\tilde{n}^{-}$is found.

Again from $5.16 n \equiv \tilde{n}^{+} \cup T \cup \tilde{n}^{-}$is a piecewise smooth manifold; we must show it separates $M$. Let $\gamma$ be an arc from $\partial U$ to $I$. Then that end of $\gamma$ near $I$ 
(i.e. in $\left.W^{\prime}\right)$ contains points on orbit segments from $\tilde{n}_{+}$to $\tilde{n}_{-}$(5.17). Let $p$ be the first such point ( $\gamma$ directed from $\partial U$ to $I$ ). If $p$ is contained in $\tilde{n}_{+}$or $\tilde{n}_{-}$we are done. If $p$ is on an orbit segment from the interior of $\tilde{n}^{+}$then it cannot be the first such point. The only remaining possibility is that $p \in T$ and so we are done.

Again the interior of $N$ is defined to be the union of the components of $N-n$ which contain $I$ which is the same as the union of the components of $U-n$ which contain $I$. The boundary of $N$ is then contained in $n=\tilde{n}^{+} \cup T \cup \tilde{n}^{-}$; it is in fact a union of components of $n$. Finally since orbits in $a^{+}$run from $\tilde{n}^{+}$to $I$ with increasing time, $\tilde{n}^{+}$actually does contain the incoming, and likewise $\tilde{n}^{-}$the outgoing, points of $N$.

This completes the proof of Theorem 1.5. An easy corollary is that if $I$, hence $a^{+}$and $a^{-}$(2.8, bottom row), has finitely generated cohomology, then $N$ can be chosen so that the inclusion induced homeomorphism $H^{*}(I) \rightarrow H^{*}(N), H^{*}\left(n^{+}\right)$ $\rightarrow H^{*}\left(a^{+}\right)$and $H^{*}\left(n^{-}\right) \rightarrow H^{*}\left(a^{-}\right)$are surjections.

\section{BIBLIOGRAPHY}

1. G. D. Birkhoff, "Nouvelles recherches sur les systèmes dynamiques," in Collected works. Vol. 2, Amer. Math. Soc., Providence, R. I., 1950, pp. 530-659.

2. C. Conley, "Invariant sets in a monkey saddle," Proceedings United States-Japan seminar on differential and functional equations, Edited by William A. Harris Jr. and Yasutaka Sibuya, Benjamin, New York, 1967. MR 36 \#5415.

3. - The retrograde circular solutions of the restricted three-body problem via a submanifold convex to the flow, SIAM J. Appl. Math. 16 (1968), 620-625. MR 37 \#3113.

4. - On the ultimate behavior of orbits with respect to an unstable critical point, J. Differential Equations 5 (1969), 136-158.

5. _-, "Twist mappings, linking, analyticity, and periodic solutions which pass close to an unstable periodic solution," Topological dynamics, Edited by Joseph Auslander, Proc. Internat. Sympos. on Topological Dynamics (Colorado State Univ., Fort Collins, Colo., 1967), Benjamin, New York, 1968.

6. C. Conley and R. Easton, "On isolated invariant sets and isolating submanifolds," in Advances in differential and integral equations, Edited by John Nohel, Benjamin, New York, pp. 97-104.

7. R. Easton, On the existence of invariant sets inside a submanifold convex to a flow, Thesis, Univ. of Wisconsin, Madison, 1967.

8. - L L L L _ Lating invariant sets, Proc. Sympos. Pure Math., vol. 14, Amer. Math. Soc., Providence, R. I., 1970, pp. 55-59.

9. - - On the existence of invariant sets inside a submanifold convex to a flow, J. Differential Equations 7 (1970), 54-68.

10. —, "A flow near a degenerate critical point," Advances in differential and integral equations, Edited by John Nohel, Benjamin, New York, p. 137.

11. J. Moser, A rapidly convergent iteration method and non-linear differential equations. II, Ann. Scuola Norm. Sup. Pisa (3) 20 (1966), 499-535. MR 34 \#6280.

12. R. Sacker, A perturbation theorem for invariant Riemannian manifolds, Differential Equations and Dynamical Systems, Proc. Internat. Sympos. (Mayaguez, P.R., 1965), Academic Press, New York, 1967, pp. 43-54. MR 36 \#1784.

13. - A new approach to the perturbation theory of invariant surfaces, Comm. Pure Appl. Math. 18 (1965), 717-732. MR 32 \#6003. 
14. S. Smale, Diffeomorphisms with many periodic points, Differential and Combinatorial Topology (A Symposium in Honor of Marston Morse), Princeton Univ. Press, Princeton, N. J., 1965, pp. 63-80. MR 31 \#6244.

15. E. H. Spanier, Algebraic topology, McGraw-Hill, New York, 1966. MR 35 \#1007.

16. T. Ura, On the flow outside a closed invariant set; stability, relative stability and saddle sets, Contributions to Differential Equations 3 (1964), 249-294. MR 29 \#321.

17. T. Ważewski, Sur une méthode topologique de l'examen de l'allure asymptotique des intégrales des équations différentielles, Proc. Internat. Congress Math. (Amsterdam, 1954) vol. III, Noordhoff, Groningen and North-Holland, Amsterdam, 1956, pp. 132-139. MR 19, 272.

\section{UNIVERSITY OF WISCONSIN,}

MADISON, WisConsin 53706 